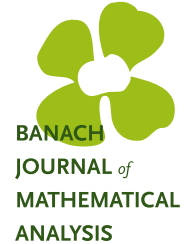

Banach J. Math. Anal. 10 (2016), no. 4, 703-726

http://dx.doi.org/10.1215/17358787-3649920

ISSN: $1735-8787$ (electronic)

http://projecteuclid.org/bjma

ANALYSIS

\title{
NONCOMMUTATIVE HARDY-LORENTZ SPACES ASSOCIATED WITH SEMIFINITE SUBDIAGONAL ALGEBRAS
}

\author{
YAZHOU HAN
}

Communicated by Q. Xu

\begin{abstract}
Let $\mathcal{A}$ be a maximal subdiagonal algebra of semifinite von Neumann algebra $\mathcal{M}$. For $0<p \leq \infty$, we define the noncommutative HardyLorentz spaces $H^{p, \omega}(\mathcal{A})$, and give some properties of these spaces. We obtain that the Herglotz maps are bounded linear maps from $\Lambda_{\omega}^{p}(\mathcal{M})$ into $\Lambda_{\omega}^{p}(\mathcal{M})$, and with this result we characterize the dual spaces of $H^{p, \omega}(\mathcal{A})$ for $1<p<\infty$. We also present the Hartman-Wintner spectral inclusion theorem of Toeplitz operators and Pisier's interpolation theorem for this case.
\end{abstract}

\section{INTRODUCTION}

Let $\mathbb{T}$ be the unit circle of a complex plane equipped with a normalized Lebesgue measure $d m$. We denote by $H^{p}(\mathbb{T})$ the usual Hardy spaces on $\mathbb{T}$, the space of functions on the unit circle which are in $L^{p}(\mathbb{T})$ with respect to the Lebesgue measure and whose negative Fourier coefficients vanish. These spaces have played an important role in modern analysis and prediction theory.

In the setting of operator algebraists, a noncommutative version of $H_{p}$ spaces was given by Arveson. In 1967, Arveson [3] introduced the concept of maximal subdiagonal algebras $\mathcal{A}$ of a von Neumann algebra $\mathcal{M}$, unifying analytic function spaces and nonself-adjoint operator algebras. In the case that $\mathcal{M}$ has a finite trace, $H^{p}(\mathcal{A})$ may be defined to be the closure of $\mathcal{A}$ in the noncommutative $L_{p}$ space $L^{p}(\mathcal{M})$. Subsequently, Arveson's pioneering work has been extended to different cases by several authors. For example, Marsalli and West [18] obtained a

Copyright 2016 by the Tusi Mathematical Research Group.

Received Aug. 10, 2015; Accepted Dec. 25, 2015.

2010 Mathematics Subject Classification. Primary 46L52; Secondary 46L51.

Keywords. subdiagonal algebras, noncommutative Hardy-Lorentz spaces, interpolation, Toeplitz operators. 


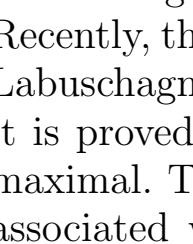

Banach J. Math. Anal. 10 (2016), no. 4, 703-726

http://dx.doi.org/10.1215/17358787-3649920

ISSN: $1735-8787$ (electronic)

http://projecteuclid.org/bjma

ANALYSIS

\title{
NONCOMMUTATIVE HARDY-LORENTZ SPACES ASSOCIATED WITH SEMIFINITE SUBDIAGONAL ALGEBRAS
}

\author{
YAZHOU HAN
}

Communicated by Q. Xu

\begin{abstract}
Let $\mathcal{A}$ be a maximal subdiagonal algebra of semifinite von Neumann algebra $\mathcal{M}$. For $0<p \leq \infty$, we define the noncommutative HardyLorentz spaces $H^{p, \omega}(\mathcal{A})$, and give some properties of these spaces. We obtain that the Herglotz maps are bounded linear maps from $\Lambda_{\omega}^{p}(\mathcal{M})$ into $\Lambda_{\omega}^{p}(\mathcal{M})$, and with this result we characterize the dual spaces of $H^{p, \omega}(\mathcal{A})$ for $1<p<\infty$. We also present the Hartman-Wintner spectral inclusion theorem of Toeplitz operators and Pisier's interpolation theorem for this case.
\end{abstract}

\section{INTRODUCTION}

Let $\mathbb{T}$ be the unit circle of a complex plane equipped with a normalized Lebesgue measure $d m$. We denote by $H^{p}(\mathbb{T})$ the usual Hardy spaces on $\mathbb{T}$, the space of functions on the unit circle which are in $L^{p}(\mathbb{T})$ with respect to the Lebesgue measure and whose negative Fourier coefficients vanish. These spaces have played an important role in modern analysis and prediction theory.

In the setting of operator algebraists, a noncommutative version of $H_{p}$ spaces was given by Arveson. In 1967, Arveson [3] introduced the concept of maximal subdiagonal algebras $\mathcal{A}$ of a von Neumann algebra $\mathcal{M}$, unifying analytic function spaces and nonself-adjoint operator algebras. In the case that $\mathcal{M}$ has a finite trace, $H^{p}(\mathcal{A})$ may be defined to be the closure of $\mathcal{A}$ in the noncommutative $L_{p}$ space $L^{p}(\mathcal{M})$. Subsequently, Arveson's pioneering work has been extended to different cases by several authors. For example, Marsalli and West [18] obtained a

Copyright 2016 by the Tusi Mathematical Research Group.

Received Aug. 10, 2015; Accepted Dec. 25, 2015.

2010 Mathematics Subject Classification. Primary 46L52; Secondary 46L51.

Keywords. subdiagonal algebras, noncommutative Hardy-Lorentz spaces, interpolation, Toeplitz operators. 


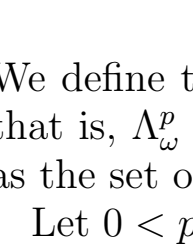

Banach J. Math. Anal. 10 (2016), no. 4, 703-726

http://dx.doi.org/10.1215/17358787-3649920

ISSN: $1735-8787$ (electronic)

http://projecteuclid.org/bjma

ANALYSIS

\title{
NONCOMMUTATIVE HARDY-LORENTZ SPACES ASSOCIATED WITH SEMIFINITE SUBDIAGONAL ALGEBRAS
}

\author{
YAZHOU HAN
}

Communicated by Q. Xu

\begin{abstract}
Let $\mathcal{A}$ be a maximal subdiagonal algebra of semifinite von Neumann algebra $\mathcal{M}$. For $0<p \leq \infty$, we define the noncommutative HardyLorentz spaces $H^{p, \omega}(\mathcal{A})$, and give some properties of these spaces. We obtain that the Herglotz maps are bounded linear maps from $\Lambda_{\omega}^{p}(\mathcal{M})$ into $\Lambda_{\omega}^{p}(\mathcal{M})$, and with this result we characterize the dual spaces of $H^{p, \omega}(\mathcal{A})$ for $1<p<\infty$. We also present the Hartman-Wintner spectral inclusion theorem of Toeplitz operators and Pisier's interpolation theorem for this case.
\end{abstract}

\section{INTRODUCTION}

Let $\mathbb{T}$ be the unit circle of a complex plane equipped with a normalized Lebesgue measure $d m$. We denote by $H^{p}(\mathbb{T})$ the usual Hardy spaces on $\mathbb{T}$, the space of functions on the unit circle which are in $L^{p}(\mathbb{T})$ with respect to the Lebesgue measure and whose negative Fourier coefficients vanish. These spaces have played an important role in modern analysis and prediction theory.

In the setting of operator algebraists, a noncommutative version of $H_{p}$ spaces was given by Arveson. In 1967, Arveson [3] introduced the concept of maximal subdiagonal algebras $\mathcal{A}$ of a von Neumann algebra $\mathcal{M}$, unifying analytic function spaces and nonself-adjoint operator algebras. In the case that $\mathcal{M}$ has a finite trace, $H^{p}(\mathcal{A})$ may be defined to be the closure of $\mathcal{A}$ in the noncommutative $L_{p}$ space $L^{p}(\mathcal{M})$. Subsequently, Arveson's pioneering work has been extended to different cases by several authors. For example, Marsalli and West [18] obtained a

Copyright 2016 by the Tusi Mathematical Research Group.

Received Aug. 10, 2015; Accepted Dec. 25, 2015.

2010 Mathematics Subject Classification. Primary 46L52; Secondary 46L51.

Keywords. subdiagonal algebras, noncommutative Hardy-Lorentz spaces, interpolation, Toeplitz operators. 


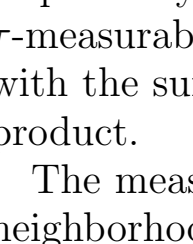

Banach J. Math. Anal. 10 (2016), no. 4, 703-726

http://dx.doi.org/10.1215/17358787-3649920

ISSN: $1735-8787$ (electronic)

http://projecteuclid.org/bjma

ANALYSIS

\title{
NONCOMMUTATIVE HARDY-LORENTZ SPACES ASSOCIATED WITH SEMIFINITE SUBDIAGONAL ALGEBRAS
}

\author{
YAZHOU HAN
}

Communicated by Q. Xu

\begin{abstract}
Let $\mathcal{A}$ be a maximal subdiagonal algebra of semifinite von Neumann algebra $\mathcal{M}$. For $0<p \leq \infty$, we define the noncommutative HardyLorentz spaces $H^{p, \omega}(\mathcal{A})$, and give some properties of these spaces. We obtain that the Herglotz maps are bounded linear maps from $\Lambda_{\omega}^{p}(\mathcal{M})$ into $\Lambda_{\omega}^{p}(\mathcal{M})$, and with this result we characterize the dual spaces of $H^{p, \omega}(\mathcal{A})$ for $1<p<\infty$. We also present the Hartman-Wintner spectral inclusion theorem of Toeplitz operators and Pisier's interpolation theorem for this case.
\end{abstract}

\section{INTRODUCTION}

Let $\mathbb{T}$ be the unit circle of a complex plane equipped with a normalized Lebesgue measure $d m$. We denote by $H^{p}(\mathbb{T})$ the usual Hardy spaces on $\mathbb{T}$, the space of functions on the unit circle which are in $L^{p}(\mathbb{T})$ with respect to the Lebesgue measure and whose negative Fourier coefficients vanish. These spaces have played an important role in modern analysis and prediction theory.

In the setting of operator algebraists, a noncommutative version of $H_{p}$ spaces was given by Arveson. In 1967, Arveson [3] introduced the concept of maximal subdiagonal algebras $\mathcal{A}$ of a von Neumann algebra $\mathcal{M}$, unifying analytic function spaces and nonself-adjoint operator algebras. In the case that $\mathcal{M}$ has a finite trace, $H^{p}(\mathcal{A})$ may be defined to be the closure of $\mathcal{A}$ in the noncommutative $L_{p}$ space $L^{p}(\mathcal{M})$. Subsequently, Arveson's pioneering work has been extended to different cases by several authors. For example, Marsalli and West [18] obtained a

Copyright 2016 by the Tusi Mathematical Research Group.

Received Aug. 10, 2015; Accepted Dec. 25, 2015.

2010 Mathematics Subject Classification. Primary 46L52; Secondary 46L51.

Keywords. subdiagonal algebras, noncommutative Hardy-Lorentz spaces, interpolation, Toeplitz operators. 


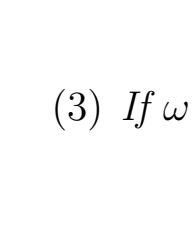

Banach J. Math. Anal. 10 (2016), no. 4, 703-726

http://dx.doi.org/10.1215/17358787-3649920

ISSN: $1735-8787$ (electronic)

http://projecteuclid.org/bjma

ANALYSIS

\title{
NONCOMMUTATIVE HARDY-LORENTZ SPACES ASSOCIATED WITH SEMIFINITE SUBDIAGONAL ALGEBRAS
}

\author{
YAZHOU HAN
}

Communicated by Q. Xu

\begin{abstract}
Let $\mathcal{A}$ be a maximal subdiagonal algebra of semifinite von Neumann algebra $\mathcal{M}$. For $0<p \leq \infty$, we define the noncommutative HardyLorentz spaces $H^{p, \omega}(\mathcal{A})$, and give some properties of these spaces. We obtain that the Herglotz maps are bounded linear maps from $\Lambda_{\omega}^{p}(\mathcal{M})$ into $\Lambda_{\omega}^{p}(\mathcal{M})$, and with this result we characterize the dual spaces of $H^{p, \omega}(\mathcal{A})$ for $1<p<\infty$. We also present the Hartman-Wintner spectral inclusion theorem of Toeplitz operators and Pisier's interpolation theorem for this case.
\end{abstract}

\section{INTRODUCTION}

Let $\mathbb{T}$ be the unit circle of a complex plane equipped with a normalized Lebesgue measure $d m$. We denote by $H^{p}(\mathbb{T})$ the usual Hardy spaces on $\mathbb{T}$, the space of functions on the unit circle which are in $L^{p}(\mathbb{T})$ with respect to the Lebesgue measure and whose negative Fourier coefficients vanish. These spaces have played an important role in modern analysis and prediction theory.

In the setting of operator algebraists, a noncommutative version of $H_{p}$ spaces was given by Arveson. In 1967, Arveson [3] introduced the concept of maximal subdiagonal algebras $\mathcal{A}$ of a von Neumann algebra $\mathcal{M}$, unifying analytic function spaces and nonself-adjoint operator algebras. In the case that $\mathcal{M}$ has a finite trace, $H^{p}(\mathcal{A})$ may be defined to be the closure of $\mathcal{A}$ in the noncommutative $L_{p}$ space $L^{p}(\mathcal{M})$. Subsequently, Arveson's pioneering work has been extended to different cases by several authors. For example, Marsalli and West [18] obtained a

Copyright 2016 by the Tusi Mathematical Research Group.

Received Aug. 10, 2015; Accepted Dec. 25, 2015.

2010 Mathematics Subject Classification. Primary 46L52; Secondary 46L51.

Keywords. subdiagonal algebras, noncommutative Hardy-Lorentz spaces, interpolation, Toeplitz operators. 


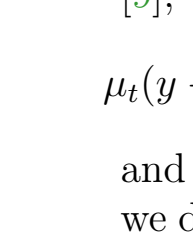

Banach J. Math. Anal. 10 (2016), no. 4, 703-726

http://dx.doi.org/10.1215/17358787-3649920

ISSN: $1735-8787$ (electronic)

http://projecteuclid.org/bjma

ANALYSIS

\title{
NONCOMMUTATIVE HARDY-LORENTZ SPACES ASSOCIATED WITH SEMIFINITE SUBDIAGONAL ALGEBRAS
}

\author{
YAZHOU HAN
}

Communicated by Q. Xu

\begin{abstract}
Let $\mathcal{A}$ be a maximal subdiagonal algebra of semifinite von Neumann algebra $\mathcal{M}$. For $0<p \leq \infty$, we define the noncommutative HardyLorentz spaces $H^{p, \omega}(\mathcal{A})$, and give some properties of these spaces. We obtain that the Herglotz maps are bounded linear maps from $\Lambda_{\omega}^{p}(\mathcal{M})$ into $\Lambda_{\omega}^{p}(\mathcal{M})$, and with this result we characterize the dual spaces of $H^{p, \omega}(\mathcal{A})$ for $1<p<\infty$. We also present the Hartman-Wintner spectral inclusion theorem of Toeplitz operators and Pisier's interpolation theorem for this case.
\end{abstract}

\section{INTRODUCTION}

Let $\mathbb{T}$ be the unit circle of a complex plane equipped with a normalized Lebesgue measure $d m$. We denote by $H^{p}(\mathbb{T})$ the usual Hardy spaces on $\mathbb{T}$, the space of functions on the unit circle which are in $L^{p}(\mathbb{T})$ with respect to the Lebesgue measure and whose negative Fourier coefficients vanish. These spaces have played an important role in modern analysis and prediction theory.

In the setting of operator algebraists, a noncommutative version of $H_{p}$ spaces was given by Arveson. In 1967, Arveson [3] introduced the concept of maximal subdiagonal algebras $\mathcal{A}$ of a von Neumann algebra $\mathcal{M}$, unifying analytic function spaces and nonself-adjoint operator algebras. In the case that $\mathcal{M}$ has a finite trace, $H^{p}(\mathcal{A})$ may be defined to be the closure of $\mathcal{A}$ in the noncommutative $L_{p}$ space $L^{p}(\mathcal{M})$. Subsequently, Arveson's pioneering work has been extended to different cases by several authors. For example, Marsalli and West [18] obtained a

Copyright 2016 by the Tusi Mathematical Research Group.

Received Aug. 10, 2015; Accepted Dec. 25, 2015.

2010 Mathematics Subject Classification. Primary 46L52; Secondary 46L51.

Keywords. subdiagonal algebras, noncommutative Hardy-Lorentz spaces, interpolation, Toeplitz operators. 


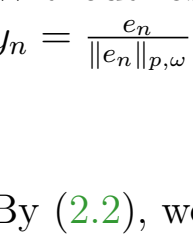

Banach J. Math. Anal. 10 (2016), no. 4, 703-726

http://dx.doi.org/10.1215/17358787-3649920

ISSN: $1735-8787$ (electronic)

http://projecteuclid.org/bjma

ANALYSIS

\title{
NONCOMMUTATIVE HARDY-LORENTZ SPACES ASSOCIATED WITH SEMIFINITE SUBDIAGONAL ALGEBRAS
}

\author{
YAZHOU HAN
}

Communicated by Q. Xu

\begin{abstract}
Let $\mathcal{A}$ be a maximal subdiagonal algebra of semifinite von Neumann algebra $\mathcal{M}$. For $0<p \leq \infty$, we define the noncommutative HardyLorentz spaces $H^{p, \omega}(\mathcal{A})$, and give some properties of these spaces. We obtain that the Herglotz maps are bounded linear maps from $\Lambda_{\omega}^{p}(\mathcal{M})$ into $\Lambda_{\omega}^{p}(\mathcal{M})$, and with this result we characterize the dual spaces of $H^{p, \omega}(\mathcal{A})$ for $1<p<\infty$. We also present the Hartman-Wintner spectral inclusion theorem of Toeplitz operators and Pisier's interpolation theorem for this case.
\end{abstract}

\section{INTRODUCTION}

Let $\mathbb{T}$ be the unit circle of a complex plane equipped with a normalized Lebesgue measure $d m$. We denote by $H^{p}(\mathbb{T})$ the usual Hardy spaces on $\mathbb{T}$, the space of functions on the unit circle which are in $L^{p}(\mathbb{T})$ with respect to the Lebesgue measure and whose negative Fourier coefficients vanish. These spaces have played an important role in modern analysis and prediction theory.

In the setting of operator algebraists, a noncommutative version of $H_{p}$ spaces was given by Arveson. In 1967, Arveson [3] introduced the concept of maximal subdiagonal algebras $\mathcal{A}$ of a von Neumann algebra $\mathcal{M}$, unifying analytic function spaces and nonself-adjoint operator algebras. In the case that $\mathcal{M}$ has a finite trace, $H^{p}(\mathcal{A})$ may be defined to be the closure of $\mathcal{A}$ in the noncommutative $L_{p}$ space $L^{p}(\mathcal{M})$. Subsequently, Arveson's pioneering work has been extended to different cases by several authors. For example, Marsalli and West [18] obtained a

Copyright 2016 by the Tusi Mathematical Research Group.

Received Aug. 10, 2015; Accepted Dec. 25, 2015.

2010 Mathematics Subject Classification. Primary 46L52; Secondary 46L51.

Keywords. subdiagonal algebras, noncommutative Hardy-Lorentz spaces, interpolation, Toeplitz operators. 


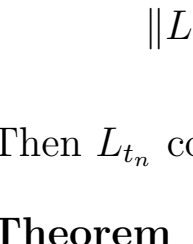

Banach J. Math. Anal. 10 (2016), no. 4, 703-726

http://dx.doi.org/10.1215/17358787-3649920

ISSN: $1735-8787$ (electronic)

http://projecteuclid.org/bjma

ANALYSIS

\title{
NONCOMMUTATIVE HARDY-LORENTZ SPACES ASSOCIATED WITH SEMIFINITE SUBDIAGONAL ALGEBRAS
}

\author{
YAZHOU HAN
}

Communicated by Q. Xu

\begin{abstract}
Let $\mathcal{A}$ be a maximal subdiagonal algebra of semifinite von Neumann algebra $\mathcal{M}$. For $0<p \leq \infty$, we define the noncommutative HardyLorentz spaces $H^{p, \omega}(\mathcal{A})$, and give some properties of these spaces. We obtain that the Herglotz maps are bounded linear maps from $\Lambda_{\omega}^{p}(\mathcal{M})$ into $\Lambda_{\omega}^{p}(\mathcal{M})$, and with this result we characterize the dual spaces of $H^{p, \omega}(\mathcal{A})$ for $1<p<\infty$. We also present the Hartman-Wintner spectral inclusion theorem of Toeplitz operators and Pisier's interpolation theorem for this case.
\end{abstract}

\section{INTRODUCTION}

Let $\mathbb{T}$ be the unit circle of a complex plane equipped with a normalized Lebesgue measure $d m$. We denote by $H^{p}(\mathbb{T})$ the usual Hardy spaces on $\mathbb{T}$, the space of functions on the unit circle which are in $L^{p}(\mathbb{T})$ with respect to the Lebesgue measure and whose negative Fourier coefficients vanish. These spaces have played an important role in modern analysis and prediction theory.

In the setting of operator algebraists, a noncommutative version of $H_{p}$ spaces was given by Arveson. In 1967, Arveson [3] introduced the concept of maximal subdiagonal algebras $\mathcal{A}$ of a von Neumann algebra $\mathcal{M}$, unifying analytic function spaces and nonself-adjoint operator algebras. In the case that $\mathcal{M}$ has a finite trace, $H^{p}(\mathcal{A})$ may be defined to be the closure of $\mathcal{A}$ in the noncommutative $L_{p}$ space $L^{p}(\mathcal{M})$. Subsequently, Arveson's pioneering work has been extended to different cases by several authors. For example, Marsalli and West [18] obtained a

Copyright 2016 by the Tusi Mathematical Research Group.

Received Aug. 10, 2015; Accepted Dec. 25, 2015.

2010 Mathematics Subject Classification. Primary 46L52; Secondary 46L51.

Keywords. subdiagonal algebras, noncommutative Hardy-Lorentz spaces, interpolation, Toeplitz operators. 


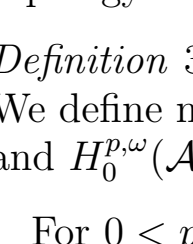

Banach J. Math. Anal. 10 (2016), no. 4, 703-726

http://dx.doi.org/10.1215/17358787-3649920

ISSN: $1735-8787$ (electronic)

http://projecteuclid.org/bjma

ANALYSIS

\title{
NONCOMMUTATIVE HARDY-LORENTZ SPACES ASSOCIATED WITH SEMIFINITE SUBDIAGONAL ALGEBRAS
}

\author{
YAZHOU HAN
}

Communicated by Q. Xu

\begin{abstract}
Let $\mathcal{A}$ be a maximal subdiagonal algebra of semifinite von Neumann algebra $\mathcal{M}$. For $0<p \leq \infty$, we define the noncommutative HardyLorentz spaces $H^{p, \omega}(\mathcal{A})$, and give some properties of these spaces. We obtain that the Herglotz maps are bounded linear maps from $\Lambda_{\omega}^{p}(\mathcal{M})$ into $\Lambda_{\omega}^{p}(\mathcal{M})$, and with this result we characterize the dual spaces of $H^{p, \omega}(\mathcal{A})$ for $1<p<\infty$. We also present the Hartman-Wintner spectral inclusion theorem of Toeplitz operators and Pisier's interpolation theorem for this case.
\end{abstract}

\section{INTRODUCTION}

Let $\mathbb{T}$ be the unit circle of a complex plane equipped with a normalized Lebesgue measure $d m$. We denote by $H^{p}(\mathbb{T})$ the usual Hardy spaces on $\mathbb{T}$, the space of functions on the unit circle which are in $L^{p}(\mathbb{T})$ with respect to the Lebesgue measure and whose negative Fourier coefficients vanish. These spaces have played an important role in modern analysis and prediction theory.

In the setting of operator algebraists, a noncommutative version of $H_{p}$ spaces was given by Arveson. In 1967, Arveson [3] introduced the concept of maximal subdiagonal algebras $\mathcal{A}$ of a von Neumann algebra $\mathcal{M}$, unifying analytic function spaces and nonself-adjoint operator algebras. In the case that $\mathcal{M}$ has a finite trace, $H^{p}(\mathcal{A})$ may be defined to be the closure of $\mathcal{A}$ in the noncommutative $L_{p}$ space $L^{p}(\mathcal{M})$. Subsequently, Arveson's pioneering work has been extended to different cases by several authors. For example, Marsalli and West [18] obtained a

Copyright 2016 by the Tusi Mathematical Research Group.

Received Aug. 10, 2015; Accepted Dec. 25, 2015.

2010 Mathematics Subject Classification. Primary 46L52; Secondary 46L51.

Keywords. subdiagonal algebras, noncommutative Hardy-Lorentz spaces, interpolation, Toeplitz operators. 


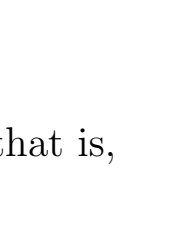

Banach J. Math. Anal. 10 (2016), no. 4, 703-726

http://dx.doi.org/10.1215/17358787-3649920

ISSN: $1735-8787$ (electronic)

http://projecteuclid.org/bjma

ANALYSIS

\title{
NONCOMMUTATIVE HARDY-LORENTZ SPACES ASSOCIATED WITH SEMIFINITE SUBDIAGONAL ALGEBRAS
}

\author{
YAZHOU HAN
}

Communicated by Q. Xu

\begin{abstract}
Let $\mathcal{A}$ be a maximal subdiagonal algebra of semifinite von Neumann algebra $\mathcal{M}$. For $0<p \leq \infty$, we define the noncommutative HardyLorentz spaces $H^{p, \omega}(\mathcal{A})$, and give some properties of these spaces. We obtain that the Herglotz maps are bounded linear maps from $\Lambda_{\omega}^{p}(\mathcal{M})$ into $\Lambda_{\omega}^{p}(\mathcal{M})$, and with this result we characterize the dual spaces of $H^{p, \omega}(\mathcal{A})$ for $1<p<\infty$. We also present the Hartman-Wintner spectral inclusion theorem of Toeplitz operators and Pisier's interpolation theorem for this case.
\end{abstract}

\section{INTRODUCTION}

Let $\mathbb{T}$ be the unit circle of a complex plane equipped with a normalized Lebesgue measure $d m$. We denote by $H^{p}(\mathbb{T})$ the usual Hardy spaces on $\mathbb{T}$, the space of functions on the unit circle which are in $L^{p}(\mathbb{T})$ with respect to the Lebesgue measure and whose negative Fourier coefficients vanish. These spaces have played an important role in modern analysis and prediction theory.

In the setting of operator algebraists, a noncommutative version of $H_{p}$ spaces was given by Arveson. In 1967, Arveson [3] introduced the concept of maximal subdiagonal algebras $\mathcal{A}$ of a von Neumann algebra $\mathcal{M}$, unifying analytic function spaces and nonself-adjoint operator algebras. In the case that $\mathcal{M}$ has a finite trace, $H^{p}(\mathcal{A})$ may be defined to be the closure of $\mathcal{A}$ in the noncommutative $L_{p}$ space $L^{p}(\mathcal{M})$. Subsequently, Arveson's pioneering work has been extended to different cases by several authors. For example, Marsalli and West [18] obtained a

Copyright 2016 by the Tusi Mathematical Research Group.

Received Aug. 10, 2015; Accepted Dec. 25, 2015.

2010 Mathematics Subject Classification. Primary 46L52; Secondary 46L51.

Keywords. subdiagonal algebras, noncommutative Hardy-Lorentz spaces, interpolation, Toeplitz operators. 


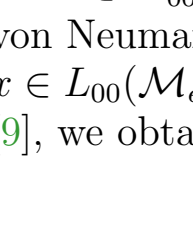

Banach J. Math. Anal. 10 (2016), no. 4, 703-726

http://dx.doi.org/10.1215/17358787-3649920

ISSN: $1735-8787$ (electronic)

http://projecteuclid.org/bjma

ANALYSIS

\title{
NONCOMMUTATIVE HARDY-LORENTZ SPACES ASSOCIATED WITH SEMIFINITE SUBDIAGONAL ALGEBRAS
}

\author{
YAZHOU HAN
}

Communicated by Q. Xu

\begin{abstract}
Let $\mathcal{A}$ be a maximal subdiagonal algebra of semifinite von Neumann algebra $\mathcal{M}$. For $0<p \leq \infty$, we define the noncommutative HardyLorentz spaces $H^{p, \omega}(\mathcal{A})$, and give some properties of these spaces. We obtain that the Herglotz maps are bounded linear maps from $\Lambda_{\omega}^{p}(\mathcal{M})$ into $\Lambda_{\omega}^{p}(\mathcal{M})$, and with this result we characterize the dual spaces of $H^{p, \omega}(\mathcal{A})$ for $1<p<\infty$. We also present the Hartman-Wintner spectral inclusion theorem of Toeplitz operators and Pisier's interpolation theorem for this case.
\end{abstract}

\section{INTRODUCTION}

Let $\mathbb{T}$ be the unit circle of a complex plane equipped with a normalized Lebesgue measure $d m$. We denote by $H^{p}(\mathbb{T})$ the usual Hardy spaces on $\mathbb{T}$, the space of functions on the unit circle which are in $L^{p}(\mathbb{T})$ with respect to the Lebesgue measure and whose negative Fourier coefficients vanish. These spaces have played an important role in modern analysis and prediction theory.

In the setting of operator algebraists, a noncommutative version of $H_{p}$ spaces was given by Arveson. In 1967, Arveson [3] introduced the concept of maximal subdiagonal algebras $\mathcal{A}$ of a von Neumann algebra $\mathcal{M}$, unifying analytic function spaces and nonself-adjoint operator algebras. In the case that $\mathcal{M}$ has a finite trace, $H^{p}(\mathcal{A})$ may be defined to be the closure of $\mathcal{A}$ in the noncommutative $L_{p}$ space $L^{p}(\mathcal{M})$. Subsequently, Arveson's pioneering work has been extended to different cases by several authors. For example, Marsalli and West [18] obtained a

Copyright 2016 by the Tusi Mathematical Research Group.

Received Aug. 10, 2015; Accepted Dec. 25, 2015.

2010 Mathematics Subject Classification. Primary 46L52; Secondary 46L51.

Keywords. subdiagonal algebras, noncommutative Hardy-Lorentz spaces, interpolation, Toeplitz operators. 


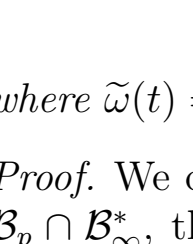

Banach J. Math. Anal. 10 (2016), no. 4, 703-726

http://dx.doi.org/10.1215/17358787-3649920

ISSN: $1735-8787$ (electronic)

http://projecteuclid.org/bjma

ANALYSIS

\title{
NONCOMMUTATIVE HARDY-LORENTZ SPACES ASSOCIATED WITH SEMIFINITE SUBDIAGONAL ALGEBRAS
}

\author{
YAZHOU HAN
}

Communicated by Q. Xu

\begin{abstract}
Let $\mathcal{A}$ be a maximal subdiagonal algebra of semifinite von Neumann algebra $\mathcal{M}$. For $0<p \leq \infty$, we define the noncommutative HardyLorentz spaces $H^{p, \omega}(\mathcal{A})$, and give some properties of these spaces. We obtain that the Herglotz maps are bounded linear maps from $\Lambda_{\omega}^{p}(\mathcal{M})$ into $\Lambda_{\omega}^{p}(\mathcal{M})$, and with this result we characterize the dual spaces of $H^{p, \omega}(\mathcal{A})$ for $1<p<\infty$. We also present the Hartman-Wintner spectral inclusion theorem of Toeplitz operators and Pisier's interpolation theorem for this case.
\end{abstract}

\section{INTRODUCTION}

Let $\mathbb{T}$ be the unit circle of a complex plane equipped with a normalized Lebesgue measure $d m$. We denote by $H^{p}(\mathbb{T})$ the usual Hardy spaces on $\mathbb{T}$, the space of functions on the unit circle which are in $L^{p}(\mathbb{T})$ with respect to the Lebesgue measure and whose negative Fourier coefficients vanish. These spaces have played an important role in modern analysis and prediction theory.

In the setting of operator algebraists, a noncommutative version of $H_{p}$ spaces was given by Arveson. In 1967, Arveson [3] introduced the concept of maximal subdiagonal algebras $\mathcal{A}$ of a von Neumann algebra $\mathcal{M}$, unifying analytic function spaces and nonself-adjoint operator algebras. In the case that $\mathcal{M}$ has a finite trace, $H^{p}(\mathcal{A})$ may be defined to be the closure of $\mathcal{A}$ in the noncommutative $L_{p}$ space $L^{p}(\mathcal{M})$. Subsequently, Arveson's pioneering work has been extended to different cases by several authors. For example, Marsalli and West [18] obtained a

Copyright 2016 by the Tusi Mathematical Research Group.

Received Aug. 10, 2015; Accepted Dec. 25, 2015.

2010 Mathematics Subject Classification. Primary 46L52; Secondary 46L51.

Keywords. subdiagonal algebras, noncommutative Hardy-Lorentz spaces, interpolation, Toeplitz operators. 


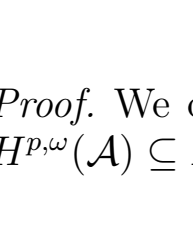

Banach J. Math. Anal. 10 (2016), no. 4, 703-726

http://dx.doi.org/10.1215/17358787-3649920

ISSN: $1735-8787$ (electronic)

http://projecteuclid.org/bjma

ANALYSIS

\title{
NONCOMMUTATIVE HARDY-LORENTZ SPACES ASSOCIATED WITH SEMIFINITE SUBDIAGONAL ALGEBRAS
}

\author{
YAZHOU HAN
}

Communicated by Q. Xu

\begin{abstract}
Let $\mathcal{A}$ be a maximal subdiagonal algebra of semifinite von Neumann algebra $\mathcal{M}$. For $0<p \leq \infty$, we define the noncommutative HardyLorentz spaces $H^{p, \omega}(\mathcal{A})$, and give some properties of these spaces. We obtain that the Herglotz maps are bounded linear maps from $\Lambda_{\omega}^{p}(\mathcal{M})$ into $\Lambda_{\omega}^{p}(\mathcal{M})$, and with this result we characterize the dual spaces of $H^{p, \omega}(\mathcal{A})$ for $1<p<\infty$. We also present the Hartman-Wintner spectral inclusion theorem of Toeplitz operators and Pisier's interpolation theorem for this case.
\end{abstract}

\section{INTRODUCTION}

Let $\mathbb{T}$ be the unit circle of a complex plane equipped with a normalized Lebesgue measure $d m$. We denote by $H^{p}(\mathbb{T})$ the usual Hardy spaces on $\mathbb{T}$, the space of functions on the unit circle which are in $L^{p}(\mathbb{T})$ with respect to the Lebesgue measure and whose negative Fourier coefficients vanish. These spaces have played an important role in modern analysis and prediction theory.

In the setting of operator algebraists, a noncommutative version of $H_{p}$ spaces was given by Arveson. In 1967, Arveson [3] introduced the concept of maximal subdiagonal algebras $\mathcal{A}$ of a von Neumann algebra $\mathcal{M}$, unifying analytic function spaces and nonself-adjoint operator algebras. In the case that $\mathcal{M}$ has a finite trace, $H^{p}(\mathcal{A})$ may be defined to be the closure of $\mathcal{A}$ in the noncommutative $L_{p}$ space $L^{p}(\mathcal{M})$. Subsequently, Arveson's pioneering work has been extended to different cases by several authors. For example, Marsalli and West [18] obtained a

Copyright 2016 by the Tusi Mathematical Research Group.

Received Aug. 10, 2015; Accepted Dec. 25, 2015.

2010 Mathematics Subject Classification. Primary 46L52; Secondary 46L51.

Keywords. subdiagonal algebras, noncommutative Hardy-Lorentz spaces, interpolation, Toeplitz operators. 


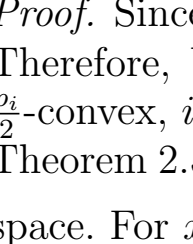

Banach J. Math. Anal. 10 (2016), no. 4, 703-726

http://dx.doi.org/10.1215/17358787-3649920

ISSN: $1735-8787$ (electronic)

http://projecteuclid.org/bjma

ANALYSIS

\title{
NONCOMMUTATIVE HARDY-LORENTZ SPACES ASSOCIATED WITH SEMIFINITE SUBDIAGONAL ALGEBRAS
}

\author{
YAZHOU HAN
}

Communicated by Q. Xu

\begin{abstract}
Let $\mathcal{A}$ be a maximal subdiagonal algebra of semifinite von Neumann algebra $\mathcal{M}$. For $0<p \leq \infty$, we define the noncommutative HardyLorentz spaces $H^{p, \omega}(\mathcal{A})$, and give some properties of these spaces. We obtain that the Herglotz maps are bounded linear maps from $\Lambda_{\omega}^{p}(\mathcal{M})$ into $\Lambda_{\omega}^{p}(\mathcal{M})$, and with this result we characterize the dual spaces of $H^{p, \omega}(\mathcal{A})$ for $1<p<\infty$. We also present the Hartman-Wintner spectral inclusion theorem of Toeplitz operators and Pisier's interpolation theorem for this case.
\end{abstract}

\section{INTRODUCTION}

Let $\mathbb{T}$ be the unit circle of a complex plane equipped with a normalized Lebesgue measure $d m$. We denote by $H^{p}(\mathbb{T})$ the usual Hardy spaces on $\mathbb{T}$, the space of functions on the unit circle which are in $L^{p}(\mathbb{T})$ with respect to the Lebesgue measure and whose negative Fourier coefficients vanish. These spaces have played an important role in modern analysis and prediction theory.

In the setting of operator algebraists, a noncommutative version of $H_{p}$ spaces was given by Arveson. In 1967, Arveson [3] introduced the concept of maximal subdiagonal algebras $\mathcal{A}$ of a von Neumann algebra $\mathcal{M}$, unifying analytic function spaces and nonself-adjoint operator algebras. In the case that $\mathcal{M}$ has a finite trace, $H^{p}(\mathcal{A})$ may be defined to be the closure of $\mathcal{A}$ in the noncommutative $L_{p}$ space $L^{p}(\mathcal{M})$. Subsequently, Arveson's pioneering work has been extended to different cases by several authors. For example, Marsalli and West [18] obtained a

Copyright 2016 by the Tusi Mathematical Research Group.

Received Aug. 10, 2015; Accepted Dec. 25, 2015.

2010 Mathematics Subject Classification. Primary 46L52; Secondary 46L51.

Keywords. subdiagonal algebras, noncommutative Hardy-Lorentz spaces, interpolation, Toeplitz operators. 


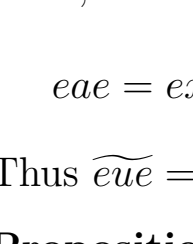

Banach J. Math. Anal. 10 (2016), no. 4, 703-726

http://dx.doi.org/10.1215/17358787-3649920

ISSN: $1735-8787$ (electronic)

http://projecteuclid.org/bjma

ANALYSIS

\title{
NONCOMMUTATIVE HARDY-LORENTZ SPACES ASSOCIATED WITH SEMIFINITE SUBDIAGONAL ALGEBRAS
}

\author{
YAZHOU HAN
}

Communicated by Q. Xu

\begin{abstract}
Let $\mathcal{A}$ be a maximal subdiagonal algebra of semifinite von Neumann algebra $\mathcal{M}$. For $0<p \leq \infty$, we define the noncommutative HardyLorentz spaces $H^{p, \omega}(\mathcal{A})$, and give some properties of these spaces. We obtain that the Herglotz maps are bounded linear maps from $\Lambda_{\omega}^{p}(\mathcal{M})$ into $\Lambda_{\omega}^{p}(\mathcal{M})$, and with this result we characterize the dual spaces of $H^{p, \omega}(\mathcal{A})$ for $1<p<\infty$. We also present the Hartman-Wintner spectral inclusion theorem of Toeplitz operators and Pisier's interpolation theorem for this case.
\end{abstract}

\section{INTRODUCTION}

Let $\mathbb{T}$ be the unit circle of a complex plane equipped with a normalized Lebesgue measure $d m$. We denote by $H^{p}(\mathbb{T})$ the usual Hardy spaces on $\mathbb{T}$, the space of functions on the unit circle which are in $L^{p}(\mathbb{T})$ with respect to the Lebesgue measure and whose negative Fourier coefficients vanish. These spaces have played an important role in modern analysis and prediction theory.

In the setting of operator algebraists, a noncommutative version of $H_{p}$ spaces was given by Arveson. In 1967, Arveson [3] introduced the concept of maximal subdiagonal algebras $\mathcal{A}$ of a von Neumann algebra $\mathcal{M}$, unifying analytic function spaces and nonself-adjoint operator algebras. In the case that $\mathcal{M}$ has a finite trace, $H^{p}(\mathcal{A})$ may be defined to be the closure of $\mathcal{A}$ in the noncommutative $L_{p}$ space $L^{p}(\mathcal{M})$. Subsequently, Arveson's pioneering work has been extended to different cases by several authors. For example, Marsalli and West [18] obtained a

Copyright 2016 by the Tusi Mathematical Research Group.

Received Aug. 10, 2015; Accepted Dec. 25, 2015.

2010 Mathematics Subject Classification. Primary 46L52; Secondary 46L51.

Keywords. subdiagonal algebras, noncommutative Hardy-Lorentz spaces, interpolation, Toeplitz operators. 


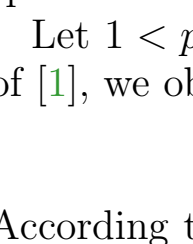

Banach J. Math. Anal. 10 (2016), no. 4, 703-726

http://dx.doi.org/10.1215/17358787-3649920

ISSN: $1735-8787$ (electronic)

http://projecteuclid.org/bjma

ANALYSIS

\title{
NONCOMMUTATIVE HARDY-LORENTZ SPACES ASSOCIATED WITH SEMIFINITE SUBDIAGONAL ALGEBRAS
}

\author{
YAZHOU HAN
}

Communicated by Q. Xu

\begin{abstract}
Let $\mathcal{A}$ be a maximal subdiagonal algebra of semifinite von Neumann algebra $\mathcal{M}$. For $0<p \leq \infty$, we define the noncommutative HardyLorentz spaces $H^{p, \omega}(\mathcal{A})$, and give some properties of these spaces. We obtain that the Herglotz maps are bounded linear maps from $\Lambda_{\omega}^{p}(\mathcal{M})$ into $\Lambda_{\omega}^{p}(\mathcal{M})$, and with this result we characterize the dual spaces of $H^{p, \omega}(\mathcal{A})$ for $1<p<\infty$. We also present the Hartman-Wintner spectral inclusion theorem of Toeplitz operators and Pisier's interpolation theorem for this case.
\end{abstract}

\section{INTRODUCTION}

Let $\mathbb{T}$ be the unit circle of a complex plane equipped with a normalized Lebesgue measure $d m$. We denote by $H^{p}(\mathbb{T})$ the usual Hardy spaces on $\mathbb{T}$, the space of functions on the unit circle which are in $L^{p}(\mathbb{T})$ with respect to the Lebesgue measure and whose negative Fourier coefficients vanish. These spaces have played an important role in modern analysis and prediction theory.

In the setting of operator algebraists, a noncommutative version of $H_{p}$ spaces was given by Arveson. In 1967, Arveson [3] introduced the concept of maximal subdiagonal algebras $\mathcal{A}$ of a von Neumann algebra $\mathcal{M}$, unifying analytic function spaces and nonself-adjoint operator algebras. In the case that $\mathcal{M}$ has a finite trace, $H^{p}(\mathcal{A})$ may be defined to be the closure of $\mathcal{A}$ in the noncommutative $L_{p}$ space $L^{p}(\mathcal{M})$. Subsequently, Arveson's pioneering work has been extended to different cases by several authors. For example, Marsalli and West [18] obtained a

Copyright 2016 by the Tusi Mathematical Research Group.

Received Aug. 10, 2015; Accepted Dec. 25, 2015.

2010 Mathematics Subject Classification. Primary 46L52; Secondary 46L51.

Keywords. subdiagonal algebras, noncommutative Hardy-Lorentz spaces, interpolation, Toeplitz operators. 


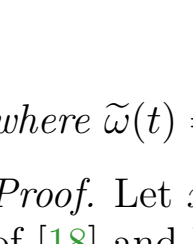

Banach J. Math. Anal. 10 (2016), no. 4, 703-726

http://dx.doi.org/10.1215/17358787-3649920

ISSN: $1735-8787$ (electronic)

http://projecteuclid.org/bjma

ANALYSIS

\title{
NONCOMMUTATIVE HARDY-LORENTZ SPACES ASSOCIATED WITH SEMIFINITE SUBDIAGONAL ALGEBRAS
}

\author{
YAZHOU HAN
}

Communicated by Q. Xu

\begin{abstract}
Let $\mathcal{A}$ be a maximal subdiagonal algebra of semifinite von Neumann algebra $\mathcal{M}$. For $0<p \leq \infty$, we define the noncommutative HardyLorentz spaces $H^{p, \omega}(\mathcal{A})$, and give some properties of these spaces. We obtain that the Herglotz maps are bounded linear maps from $\Lambda_{\omega}^{p}(\mathcal{M})$ into $\Lambda_{\omega}^{p}(\mathcal{M})$, and with this result we characterize the dual spaces of $H^{p, \omega}(\mathcal{A})$ for $1<p<\infty$. We also present the Hartman-Wintner spectral inclusion theorem of Toeplitz operators and Pisier's interpolation theorem for this case.
\end{abstract}

\section{INTRODUCTION}

Let $\mathbb{T}$ be the unit circle of a complex plane equipped with a normalized Lebesgue measure $d m$. We denote by $H^{p}(\mathbb{T})$ the usual Hardy spaces on $\mathbb{T}$, the space of functions on the unit circle which are in $L^{p}(\mathbb{T})$ with respect to the Lebesgue measure and whose negative Fourier coefficients vanish. These spaces have played an important role in modern analysis and prediction theory.

In the setting of operator algebraists, a noncommutative version of $H_{p}$ spaces was given by Arveson. In 1967, Arveson [3] introduced the concept of maximal subdiagonal algebras $\mathcal{A}$ of a von Neumann algebra $\mathcal{M}$, unifying analytic function spaces and nonself-adjoint operator algebras. In the case that $\mathcal{M}$ has a finite trace, $H^{p}(\mathcal{A})$ may be defined to be the closure of $\mathcal{A}$ in the noncommutative $L_{p}$ space $L^{p}(\mathcal{M})$. Subsequently, Arveson's pioneering work has been extended to different cases by several authors. For example, Marsalli and West [18] obtained a

Copyright 2016 by the Tusi Mathematical Research Group.

Received Aug. 10, 2015; Accepted Dec. 25, 2015.

2010 Mathematics Subject Classification. Primary 46L52; Secondary 46L51.

Keywords. subdiagonal algebras, noncommutative Hardy-Lorentz spaces, interpolation, Toeplitz operators. 


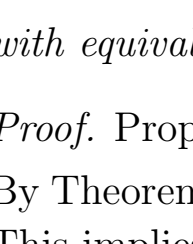

Banach J. Math. Anal. 10 (2016), no. 4, 703-726

http://dx.doi.org/10.1215/17358787-3649920

ISSN: $1735-8787$ (electronic)

http://projecteuclid.org/bjma

ANALYSIS

\title{
NONCOMMUTATIVE HARDY-LORENTZ SPACES ASSOCIATED WITH SEMIFINITE SUBDIAGONAL ALGEBRAS
}

\author{
YAZHOU HAN
}

Communicated by Q. Xu

\begin{abstract}
Let $\mathcal{A}$ be a maximal subdiagonal algebra of semifinite von Neumann algebra $\mathcal{M}$. For $0<p \leq \infty$, we define the noncommutative HardyLorentz spaces $H^{p, \omega}(\mathcal{A})$, and give some properties of these spaces. We obtain that the Herglotz maps are bounded linear maps from $\Lambda_{\omega}^{p}(\mathcal{M})$ into $\Lambda_{\omega}^{p}(\mathcal{M})$, and with this result we characterize the dual spaces of $H^{p, \omega}(\mathcal{A})$ for $1<p<\infty$. We also present the Hartman-Wintner spectral inclusion theorem of Toeplitz operators and Pisier's interpolation theorem for this case.
\end{abstract}

\section{INTRODUCTION}

Let $\mathbb{T}$ be the unit circle of a complex plane equipped with a normalized Lebesgue measure $d m$. We denote by $H^{p}(\mathbb{T})$ the usual Hardy spaces on $\mathbb{T}$, the space of functions on the unit circle which are in $L^{p}(\mathbb{T})$ with respect to the Lebesgue measure and whose negative Fourier coefficients vanish. These spaces have played an important role in modern analysis and prediction theory.

In the setting of operator algebraists, a noncommutative version of $H_{p}$ spaces was given by Arveson. In 1967, Arveson [3] introduced the concept of maximal subdiagonal algebras $\mathcal{A}$ of a von Neumann algebra $\mathcal{M}$, unifying analytic function spaces and nonself-adjoint operator algebras. In the case that $\mathcal{M}$ has a finite trace, $H^{p}(\mathcal{A})$ may be defined to be the closure of $\mathcal{A}$ in the noncommutative $L_{p}$ space $L^{p}(\mathcal{M})$. Subsequently, Arveson's pioneering work has been extended to different cases by several authors. For example, Marsalli and West [18] obtained a

Copyright 2016 by the Tusi Mathematical Research Group.

Received Aug. 10, 2015; Accepted Dec. 25, 2015.

2010 Mathematics Subject Classification. Primary 46L52; Secondary 46L51.

Keywords. subdiagonal algebras, noncommutative Hardy-Lorentz spaces, interpolation, Toeplitz operators. 


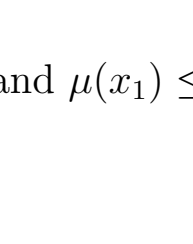

Banach J. Math. Anal. 10 (2016), no. 4, 703-726

http://dx.doi.org/10.1215/17358787-3649920

ISSN: $1735-8787$ (electronic)

http://projecteuclid.org/bjma

ANALYSIS

\title{
NONCOMMUTATIVE HARDY-LORENTZ SPACES ASSOCIATED WITH SEMIFINITE SUBDIAGONAL ALGEBRAS
}

\author{
YAZHOU HAN
}

Communicated by Q. Xu

\begin{abstract}
Let $\mathcal{A}$ be a maximal subdiagonal algebra of semifinite von Neumann algebra $\mathcal{M}$. For $0<p \leq \infty$, we define the noncommutative HardyLorentz spaces $H^{p, \omega}(\mathcal{A})$, and give some properties of these spaces. We obtain that the Herglotz maps are bounded linear maps from $\Lambda_{\omega}^{p}(\mathcal{M})$ into $\Lambda_{\omega}^{p}(\mathcal{M})$, and with this result we characterize the dual spaces of $H^{p, \omega}(\mathcal{A})$ for $1<p<\infty$. We also present the Hartman-Wintner spectral inclusion theorem of Toeplitz operators and Pisier's interpolation theorem for this case.
\end{abstract}

\section{INTRODUCTION}

Let $\mathbb{T}$ be the unit circle of a complex plane equipped with a normalized Lebesgue measure $d m$. We denote by $H^{p}(\mathbb{T})$ the usual Hardy spaces on $\mathbb{T}$, the space of functions on the unit circle which are in $L^{p}(\mathbb{T})$ with respect to the Lebesgue measure and whose negative Fourier coefficients vanish. These spaces have played an important role in modern analysis and prediction theory.

In the setting of operator algebraists, a noncommutative version of $H_{p}$ spaces was given by Arveson. In 1967, Arveson [3] introduced the concept of maximal subdiagonal algebras $\mathcal{A}$ of a von Neumann algebra $\mathcal{M}$, unifying analytic function spaces and nonself-adjoint operator algebras. In the case that $\mathcal{M}$ has a finite trace, $H^{p}(\mathcal{A})$ may be defined to be the closure of $\mathcal{A}$ in the noncommutative $L_{p}$ space $L^{p}(\mathcal{M})$. Subsequently, Arveson's pioneering work has been extended to different cases by several authors. For example, Marsalli and West [18] obtained a

Copyright 2016 by the Tusi Mathematical Research Group.

Received Aug. 10, 2015; Accepted Dec. 25, 2015.

2010 Mathematics Subject Classification. Primary 46L52; Secondary 46L51.

Keywords. subdiagonal algebras, noncommutative Hardy-Lorentz spaces, interpolation, Toeplitz operators. 


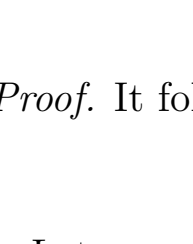

Banach J. Math. Anal. 10 (2016), no. 4, 703-726

http://dx.doi.org/10.1215/17358787-3649920

ISSN: $1735-8787$ (electronic)

http://projecteuclid.org/bjma

ANALYSIS

\title{
NONCOMMUTATIVE HARDY-LORENTZ SPACES ASSOCIATED WITH SEMIFINITE SUBDIAGONAL ALGEBRAS
}

\author{
YAZHOU HAN
}

Communicated by Q. Xu

\begin{abstract}
Let $\mathcal{A}$ be a maximal subdiagonal algebra of semifinite von Neumann algebra $\mathcal{M}$. For $0<p \leq \infty$, we define the noncommutative HardyLorentz spaces $H^{p, \omega}(\mathcal{A})$, and give some properties of these spaces. We obtain that the Herglotz maps are bounded linear maps from $\Lambda_{\omega}^{p}(\mathcal{M})$ into $\Lambda_{\omega}^{p}(\mathcal{M})$, and with this result we characterize the dual spaces of $H^{p, \omega}(\mathcal{A})$ for $1<p<\infty$. We also present the Hartman-Wintner spectral inclusion theorem of Toeplitz operators and Pisier's interpolation theorem for this case.
\end{abstract}

\section{INTRODUCTION}

Let $\mathbb{T}$ be the unit circle of a complex plane equipped with a normalized Lebesgue measure $d m$. We denote by $H^{p}(\mathbb{T})$ the usual Hardy spaces on $\mathbb{T}$, the space of functions on the unit circle which are in $L^{p}(\mathbb{T})$ with respect to the Lebesgue measure and whose negative Fourier coefficients vanish. These spaces have played an important role in modern analysis and prediction theory.

In the setting of operator algebraists, a noncommutative version of $H_{p}$ spaces was given by Arveson. In 1967, Arveson [3] introduced the concept of maximal subdiagonal algebras $\mathcal{A}$ of a von Neumann algebra $\mathcal{M}$, unifying analytic function spaces and nonself-adjoint operator algebras. In the case that $\mathcal{M}$ has a finite trace, $H^{p}(\mathcal{A})$ may be defined to be the closure of $\mathcal{A}$ in the noncommutative $L_{p}$ space $L^{p}(\mathcal{M})$. Subsequently, Arveson's pioneering work has been extended to different cases by several authors. For example, Marsalli and West [18] obtained a

Copyright 2016 by the Tusi Mathematical Research Group.

Received Aug. 10, 2015; Accepted Dec. 25, 2015.

2010 Mathematics Subject Classification. Primary 46L52; Secondary 46L51.

Keywords. subdiagonal algebras, noncommutative Hardy-Lorentz spaces, interpolation, Toeplitz operators. 


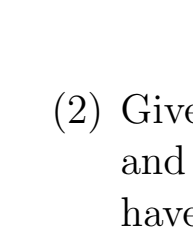

Banach J. Math. Anal. 10 (2016), no. 4, 703-726

http://dx.doi.org/10.1215/17358787-3649920

ISSN: $1735-8787$ (electronic)

http://projecteuclid.org/bjma

ANALYSIS

\title{
NONCOMMUTATIVE HARDY-LORENTZ SPACES ASSOCIATED WITH SEMIFINITE SUBDIAGONAL ALGEBRAS
}

\author{
YAZHOU HAN
}

Communicated by Q. Xu

\begin{abstract}
Let $\mathcal{A}$ be a maximal subdiagonal algebra of semifinite von Neumann algebra $\mathcal{M}$. For $0<p \leq \infty$, we define the noncommutative HardyLorentz spaces $H^{p, \omega}(\mathcal{A})$, and give some properties of these spaces. We obtain that the Herglotz maps are bounded linear maps from $\Lambda_{\omega}^{p}(\mathcal{M})$ into $\Lambda_{\omega}^{p}(\mathcal{M})$, and with this result we characterize the dual spaces of $H^{p, \omega}(\mathcal{A})$ for $1<p<\infty$. We also present the Hartman-Wintner spectral inclusion theorem of Toeplitz operators and Pisier's interpolation theorem for this case.
\end{abstract}

\section{INTRODUCTION}

Let $\mathbb{T}$ be the unit circle of a complex plane equipped with a normalized Lebesgue measure $d m$. We denote by $H^{p}(\mathbb{T})$ the usual Hardy spaces on $\mathbb{T}$, the space of functions on the unit circle which are in $L^{p}(\mathbb{T})$ with respect to the Lebesgue measure and whose negative Fourier coefficients vanish. These spaces have played an important role in modern analysis and prediction theory.

In the setting of operator algebraists, a noncommutative version of $H_{p}$ spaces was given by Arveson. In 1967, Arveson [3] introduced the concept of maximal subdiagonal algebras $\mathcal{A}$ of a von Neumann algebra $\mathcal{M}$, unifying analytic function spaces and nonself-adjoint operator algebras. In the case that $\mathcal{M}$ has a finite trace, $H^{p}(\mathcal{A})$ may be defined to be the closure of $\mathcal{A}$ in the noncommutative $L_{p}$ space $L^{p}(\mathcal{M})$. Subsequently, Arveson's pioneering work has been extended to different cases by several authors. For example, Marsalli and West [18] obtained a

Copyright 2016 by the Tusi Mathematical Research Group.

Received Aug. 10, 2015; Accepted Dec. 25, 2015.

2010 Mathematics Subject Classification. Primary 46L52; Secondary 46L51.

Keywords. subdiagonal algebras, noncommutative Hardy-Lorentz spaces, interpolation, Toeplitz operators. 


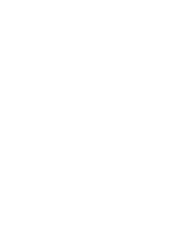

Banach J. Math. Anal. 10 (2016), no. 4, 703-726

http://dx.doi.org/10.1215/17358787-3649920

ISSN: $1735-8787$ (electronic)

http://projecteuclid.org/bjma

ANALYSIS

\title{
NONCOMMUTATIVE HARDY-LORENTZ SPACES ASSOCIATED WITH SEMIFINITE SUBDIAGONAL ALGEBRAS
}

\author{
YAZHOU HAN
}

Communicated by Q. Xu

\begin{abstract}
Let $\mathcal{A}$ be a maximal subdiagonal algebra of semifinite von Neumann algebra $\mathcal{M}$. For $0<p \leq \infty$, we define the noncommutative HardyLorentz spaces $H^{p, \omega}(\mathcal{A})$, and give some properties of these spaces. We obtain that the Herglotz maps are bounded linear maps from $\Lambda_{\omega}^{p}(\mathcal{M})$ into $\Lambda_{\omega}^{p}(\mathcal{M})$, and with this result we characterize the dual spaces of $H^{p, \omega}(\mathcal{A})$ for $1<p<\infty$. We also present the Hartman-Wintner spectral inclusion theorem of Toeplitz operators and Pisier's interpolation theorem for this case.
\end{abstract}

\section{INTRODUCTION}

Let $\mathbb{T}$ be the unit circle of a complex plane equipped with a normalized Lebesgue measure $d m$. We denote by $H^{p}(\mathbb{T})$ the usual Hardy spaces on $\mathbb{T}$, the space of functions on the unit circle which are in $L^{p}(\mathbb{T})$ with respect to the Lebesgue measure and whose negative Fourier coefficients vanish. These spaces have played an important role in modern analysis and prediction theory.

In the setting of operator algebraists, a noncommutative version of $H_{p}$ spaces was given by Arveson. In 1967, Arveson [3] introduced the concept of maximal subdiagonal algebras $\mathcal{A}$ of a von Neumann algebra $\mathcal{M}$, unifying analytic function spaces and nonself-adjoint operator algebras. In the case that $\mathcal{M}$ has a finite trace, $H^{p}(\mathcal{A})$ may be defined to be the closure of $\mathcal{A}$ in the noncommutative $L_{p}$ space $L^{p}(\mathcal{M})$. Subsequently, Arveson's pioneering work has been extended to different cases by several authors. For example, Marsalli and West [18] obtained a

Copyright 2016 by the Tusi Mathematical Research Group.

Received Aug. 10, 2015; Accepted Dec. 25, 2015.

2010 Mathematics Subject Classification. Primary 46L52; Secondary 46L51.

Keywords. subdiagonal algebras, noncommutative Hardy-Lorentz spaces, interpolation, Toeplitz operators. 


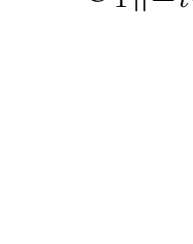

Banach J. Math. Anal. 10 (2016), no. 4, 703-726

http://dx.doi.org/10.1215/17358787-3649920

ISSN: $1735-8787$ (electronic)

http://projecteuclid.org/bjma

ANALYSIS

\title{
NONCOMMUTATIVE HARDY-LORENTZ SPACES ASSOCIATED WITH SEMIFINITE SUBDIAGONAL ALGEBRAS
}

\author{
YAZHOU HAN
}

Communicated by Q. Xu

\begin{abstract}
Let $\mathcal{A}$ be a maximal subdiagonal algebra of semifinite von Neumann algebra $\mathcal{M}$. For $0<p \leq \infty$, we define the noncommutative HardyLorentz spaces $H^{p, \omega}(\mathcal{A})$, and give some properties of these spaces. We obtain that the Herglotz maps are bounded linear maps from $\Lambda_{\omega}^{p}(\mathcal{M})$ into $\Lambda_{\omega}^{p}(\mathcal{M})$, and with this result we characterize the dual spaces of $H^{p, \omega}(\mathcal{A})$ for $1<p<\infty$. We also present the Hartman-Wintner spectral inclusion theorem of Toeplitz operators and Pisier's interpolation theorem for this case.
\end{abstract}

\section{INTRODUCTION}

Let $\mathbb{T}$ be the unit circle of a complex plane equipped with a normalized Lebesgue measure $d m$. We denote by $H^{p}(\mathbb{T})$ the usual Hardy spaces on $\mathbb{T}$, the space of functions on the unit circle which are in $L^{p}(\mathbb{T})$ with respect to the Lebesgue measure and whose negative Fourier coefficients vanish. These spaces have played an important role in modern analysis and prediction theory.

In the setting of operator algebraists, a noncommutative version of $H_{p}$ spaces was given by Arveson. In 1967, Arveson [3] introduced the concept of maximal subdiagonal algebras $\mathcal{A}$ of a von Neumann algebra $\mathcal{M}$, unifying analytic function spaces and nonself-adjoint operator algebras. In the case that $\mathcal{M}$ has a finite trace, $H^{p}(\mathcal{A})$ may be defined to be the closure of $\mathcal{A}$ in the noncommutative $L_{p}$ space $L^{p}(\mathcal{M})$. Subsequently, Arveson's pioneering work has been extended to different cases by several authors. For example, Marsalli and West [18] obtained a

Copyright 2016 by the Tusi Mathematical Research Group.

Received Aug. 10, 2015; Accepted Dec. 25, 2015.

2010 Mathematics Subject Classification. Primary 46L52; Secondary 46L51.

Keywords. subdiagonal algebras, noncommutative Hardy-Lorentz spaces, interpolation, Toeplitz operators. 


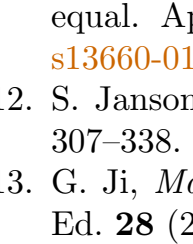

Banach J. Math. Anal. 10 (2016), no. 4, 703-726

http://dx.doi.org/10.1215/17358787-3649920

ISSN: $1735-8787$ (electronic)

http://projecteuclid.org/bjma

ANALYSIS

\title{
NONCOMMUTATIVE HARDY-LORENTZ SPACES ASSOCIATED WITH SEMIFINITE SUBDIAGONAL ALGEBRAS
}

\author{
YAZHOU HAN
}

Communicated by Q. Xu

\begin{abstract}
Let $\mathcal{A}$ be a maximal subdiagonal algebra of semifinite von Neumann algebra $\mathcal{M}$. For $0<p \leq \infty$, we define the noncommutative HardyLorentz spaces $H^{p, \omega}(\mathcal{A})$, and give some properties of these spaces. We obtain that the Herglotz maps are bounded linear maps from $\Lambda_{\omega}^{p}(\mathcal{M})$ into $\Lambda_{\omega}^{p}(\mathcal{M})$, and with this result we characterize the dual spaces of $H^{p, \omega}(\mathcal{A})$ for $1<p<\infty$. We also present the Hartman-Wintner spectral inclusion theorem of Toeplitz operators and Pisier's interpolation theorem for this case.
\end{abstract}

\section{INTRODUCTION}

Let $\mathbb{T}$ be the unit circle of a complex plane equipped with a normalized Lebesgue measure $d m$. We denote by $H^{p}(\mathbb{T})$ the usual Hardy spaces on $\mathbb{T}$, the space of functions on the unit circle which are in $L^{p}(\mathbb{T})$ with respect to the Lebesgue measure and whose negative Fourier coefficients vanish. These spaces have played an important role in modern analysis and prediction theory.

In the setting of operator algebraists, a noncommutative version of $H_{p}$ spaces was given by Arveson. In 1967, Arveson [3] introduced the concept of maximal subdiagonal algebras $\mathcal{A}$ of a von Neumann algebra $\mathcal{M}$, unifying analytic function spaces and nonself-adjoint operator algebras. In the case that $\mathcal{M}$ has a finite trace, $H^{p}(\mathcal{A})$ may be defined to be the closure of $\mathcal{A}$ in the noncommutative $L_{p}$ space $L^{p}(\mathcal{M})$. Subsequently, Arveson's pioneering work has been extended to different cases by several authors. For example, Marsalli and West [18] obtained a

Copyright 2016 by the Tusi Mathematical Research Group.

Received Aug. 10, 2015; Accepted Dec. 25, 2015.

2010 Mathematics Subject Classification. Primary 46L52; Secondary 46L51.

Keywords. subdiagonal algebras, noncommutative Hardy-Lorentz spaces, interpolation, Toeplitz operators. 